\title{
Método Simplificado para Manutenção da Concentração Sangüínea de Propofol em Nível Aproximadamente Constante, Associado ao Óxido Nitroso no Paciente Pediátrico *
}

\author{
Simplified Method to Maintain Propofol Blood Concentration in an \\ Approximately Constant Level Associated to Nitrous Oxide in \\ Pediatric Patients
}

Pedro Thadeu Galvão Vianna, TSA ${ }^{1}$, Elizabeth Pricoli Vilela ${ }^{2}$, Francisco Carlos Obata Cordon ${ }^{2}$, Lídia Raquel de Carvalho ${ }^{3}$

\section{RESUMO}

Vianna PTG, Vilela EP, Cordon FCO, Carvalho LR - Método Simplificado para Manutenção da Concentração Sangüínea de Propofol em Nível Aproximadamente Constante, Associado ao Óxido Nitroso no Paciente Pediátrico

\begin{abstract}
Justificativa e Objetivos - A manutenção de concentração sangüínea alvo-controlada em níveis aproximadamente constantes do propofol é uma técnica que pode ser empregada de modo simplificado na sala de cirurgia. A finalidade desta pesquisa é comparar clínica e laboratorialmente a infusão de propofol em crianças usando os atributos farmacocinéticos de Short e de Marsh.

Método - Foram estudados 41 pacientes com a idade de 4 a 12 anos, de ambos os sexos, estado físico ASA I ou II, distribuídos em dois grupos $S$ (20 pacientes) e M (21 pacientes). No Grupo $S$ utilizaram-se os atributos farmacocinéticos de Short, e no Grupo M, os atributos farmacocinéticos de Marsh. A indução anestésica foi feita com bolus de alfentanil $30 \mu \mathrm{g} \cdot \mathrm{kg}^{-1}$, propofol $3 \mathrm{mg} \cdot \mathrm{kg}^{-1}$ e pancurônio, $0,08 \mathrm{mg} \cdot \mathrm{kg}^{-1}$ por via venosa. Procedeu-se a intubação traqueal e a manutenção com $\mathrm{N}_{2} \mathrm{O} / \mathrm{O}_{2}$ (60\%) em ventilação controlada mecânica. No grupo $S$ a infusão de propofol foi de 254 (30 min) seguido de 216 $\mu \mathrm{g} \cdot \mathrm{kg}^{-1} \cdot \mathrm{min}^{-1}$ pormais $30 \mathrm{~min}$. No grupo $\mathrm{M}$ a infusão de propofol foi de 208 (30 min) seguido de $170 \mu \mathrm{g} \cdot \mathrm{kg}^{-1} \cdot \mathrm{min}^{-1}$ por mais 30 min. Através do atributo farmacocinético específico a cada grupo a meta foi a obtenção da concentração-alvo de $4 \mu \mathrm{g} \cdot \mathrm{kg}^{-1}$ de propofol. Foram colhidas três amostras sangüíneas (aos 20, 40 e 60 minutos) para a dosagem do propofol pelo método da Cromatografia Líquida de Alta Performance.
\end{abstract}

Resultados - Os Grupos S e M foram considerados similares quanto à idade, altura, peso e sexo $(p>0,05)$. Não houve diferença estatística significativa entre os dois grupos

\footnotetext{
${ }^{*}$ Recebido do (Received from) CET/SBA do Departamento de Anestesiologia da Faculdade de Medicina de Botucatu (FMB - UNESP). Trabalho financiado pela FAPESP

1. Professor Titular do CET/SBA do Departamento de Anestesiologia da FMB - UNESP

2. ME do CET/SBA da FMB - UNESP

3. Professora Assistente Doutora do Departamento de Bioestatística do Instituto de Biociências da FMB - UNESP
}

Apresentado (Submitted) em 15 de agosto de 2001

Aceito (Accepted) para publicação em 06 de novembro de 2001

Correspondência para (Mail to):

Dr. Pedro Thadeu Galvão Vianna

Dept ${ }^{\circ}$ de Anestesiologia da FMB - UNESP

Distrito de Rubião Junior

18618-970 Botucatu, SP

E-mail:ptgv@uol.com.br

(c) Sociedade Brasileira de Anestesiologia, 2002 estudados para os parâmetros: PAS, PAD, FC, $\mathrm{FiN}_{2} \mathrm{O}, \mathrm{SpO}_{2}$ da hemoglobina e $P_{E T} C_{2}$ no final da expiração. A comparação entre grupos no número de bolus repetidos de alfentanil não foi estatisticamente significativa. $O$ índice bispectral (BIS) não apresentou diferença estatisticamente significativa entre $M_{0}$ (vigília) e os demais momentos em ambos os grupos. Os valores Medianos da Performance do Erro (MPE) e os valores Medianos Absolutos da Performance do Erro (MAPE) mostraram diferenças estatísticas significativas entre os grupos no momento 60. Valores medianos da concentração sangüínea de propofol $\left(\mu \mathrm{g} \cdot \mathrm{kg}^{-1}\right)$ mostraram diferenças estatísticas significativas entre Me S no momento 60 e entre os momentos 40 e 60 no grupo $S$.

Conclusões - A anestesia com propofol usando os atributos farmacocinéticos de Marsh (Grupo M) apresentou menor erro no cálculo da concentração-alvo de propofol de $4 \mu \mathrm{g} \cdot \mathrm{kg}^{-1}$. Além disso, utiliza menor quantidade de propofol para obter resultados clínicos semelhantes. Por todas essas qualidades deve ser a preferida para uso em crianças ASA I e com idades entre 4 e 12 anos.

UNITERMOS - ANESTÉSICOS, Gasoso: óxido nitroso; HIPNÓTICOS: propofol; TÉCNICAS ANESTÉSICAS, Venosa: anestesia venosa

\section{SUMMARY}

Vianna PTG, Vilela EP, Cordon FCO, Carvalho LR - Simplified Method to Maintain Propofol Blood Concentration in an Approximately Constant Level Associated to Nitrous Oxide in Pediatric Patients

Background and Objectives - Maintaining target-controlled propofol blood concentrations in approximately constant levels is a technique that can be used in a simple way in the operating room. The aim of this study was to compare in clinical and laboratorial terms propofol infusion in children, using Short's and Marsh's pharmacokinetic parameters.

Methods - Forty-one patients of both genders, aged 4 to 12 years, physical status ASAl or ASAll were distributed in two groups: Group $S(n=20)$ and Group $M(n=21)$. Short's pharmacokinetic parameters were applied to group $S$, while Marsh's pharmacokinetic parameters were applied to group $M$. Intravenous anesthesia was induced with $30 \mu \mathrm{g}^{\mathrm{kg}} \mathrm{g}^{-1}$ bolus alfentanil, $3 \mathrm{mg} \cdot \mathrm{kg}^{-1}$ propofol and $0.08 \mathrm{mg} \cdot \mathrm{kg}^{-1}$ pancuronium. Patients were intubated and anesthesia was maintained with $\mathrm{N}_{2} \mathrm{O} / \mathrm{O}_{2}(60 \%)$ in controlled mechanical ventilation. Propofol infusion in group $S$ was $254 \mu \mathrm{g} \cdot \mathrm{kg}^{-1}$ (30 min) followed by 216 $\mu \mathrm{g} \cdot \mathrm{kg}^{-1} \cdot \mathrm{min}^{-1}$ for additional 30 minutes. Propofol infusion in group $\mathrm{M}$ was $208 \mu \mathrm{g} \cdot \mathrm{kg}^{-1}$ (30 min.) followed by $170 \mu \mathrm{g} \cdot \mathrm{kg}^{-1} \cdot \mathrm{min}^{-1}$ for additional 30 minutes. Using specific pharmacokinetic parameters for each group, the goal was a target-concentration of $4 \mu \mathrm{g} . \mathrm{kg}^{-1}$ propofol. Three blood samples were collected (at 20, 40 and 60 minutes) to measure propofol by the High Performance Liquid Chromatography method. 
Results - Groups $S$ and $M$ were similar in age, height, weight and gender $(p>0.05)$. There were no statistically significant differences between groups in SBP, DBP, HR, FiN $\mathrm{N}_{2} \mathrm{O}$, hemoglobin $\mathrm{SpO}_{2}$ and end tidal $\mathrm{P}_{E T} \mathrm{CO}_{2}$. The number of repeated alfentanil boluses showed no statistically significant difference between both groups. Bispectral index (BIS) showed also no statistically significant differences between MO (awaken) and remaining moments in both groups. Error Performance Median (EPM) and Error Performance Absolute Median (EPAM) values were statistically different between groups in moment 60. Median propofol blood concentrations $\left(\mu \mathrm{g} . \mathrm{kg}^{-1}\right)$ were significantly different between groups $M$ and $S$ in moment 60 and between moments 40 and 60 in group $S$.

Conclusions - Anesthesia with propofol using Marsh's pharmacokinetic parameters (group M) showed a lower error rate for obtaining $4 \mu \mathrm{g} \cdot \mathrm{kg}^{-1}$ propofol target-concentration. In addition, less propofol was needed to obtain similar clinical results. For these reasons, it should be the method of choice for children ASA I aged 4 to 12 years.

KEY WORDS - ANESTHETICS: Gaseous: nitrous oxide; ANESTHETIC TECHNIQUES, Venous: intravenous anesthesia; HYPNOTICS: propofol

\section{INTRODUÇÃO}

K ruger-Thiemer ${ }^{1}$ equacionou um modo de fornecer o ritmo de infusão de uma droga em função do tempo, coma finalidade de alcançar e manter constante a sua concentração no compartimento central, desde que sua farmacocinética pudesse ser descrita por modelo multicompartimental linear ${ }^{2,3}$. O trabalho desse autor ${ }^{1}$, desenvolvido na prática clínica para infusão venosa contínua, teve crescimento a partir da década de setenta. Entretanto, sua introdução comercial é mais recente, pois data da década de noventa ${ }^{4}$. A liberação de drogas é definida como automática quando um instrumento mecânico ou eletrônico faz os ajustes necessários de doses, independentemente da intervenção humana (manual). Os microcomputadores, com aceitação cada vez maior em Medicina, possibilitaram a realização dessa liberação automática de fármacos. Assim, por meio do modelo farmacocinético de determinado anestésico, desenvolvido por programa de computação, provê-se recurso para sua administração de acordo com a concentração desejada no plasma $^{5}$. Da mesma forma, consegue-se determinar em que tempo, após o término do fornecimento do anestésico venoso, pode-se obter a concentração em que há recuperação da consciência.

O sucesso dessa modalidade de administração de agentes anestésicos depende em grande parte do menor custo operacional do procedimento anestésico. Para esse tipo de infusão, haveria a necessidade de utilização de bombas de infusão controladas por computador ou sistema CACI-Computerized Assisted Continuous Infusion ${ }^{4,6-8}$, o que não é possível para a maioria dos atos anestésicos.

Em 1993 Bailey ${ }^{9}$ desenvolveu uma aproximação dessa infusão, que varia constantemente, entre duas ou mais taxas constantes de administração venosa contínua, taxas que decrescem de modo controlado. Com isso, ele descreveu teoricamente uma técnica para o cálculo do ritmo de infusões se- qüenciais necessárias para se aproximar do nível sangüíneo constante.

Este método foi adaptado por Vianna ${ }^{10-12}$, para uso do propofol em infusão contínua ${ }^{11}$, utilizando-se os atributos farmacocinéticos obtidos por Marsh ${ }^{13}$ e por Short ${ }^{14}$, tendo como objetivo popularizar o uso da concentração-alvo controlada, ou seja, a manutenção das concentrações sangüíneas desejadas em níveis aproximadamente constantes.

Afinalidade desta pesquisa é comparar clínica e laboratorialmente os atributos farmacocinéticos de Marsh ${ }^{13}$ e Short ${ }^{14}$, usados para controle-alvo do propofol associado ao óxido nitroso, em crianças com idades entre 4 e 12 anos.

\section{MÉTODO}

Após aprovação pela Comissão de Ética da Faculdade de Medicina de Botucatu foram estudados 41 pacientes pediátricos, de ambos os sexos, com idades entre 4 e 12 anos, pesos variáveis, em bom estado geral, estado físico ASA I, que foram submetidos à anestesia geral para cirurgias de adenoamigdalectomia, correção de estrabismo e reduções cruentas de fraturas ósseas. Os pacientes foram internados na véspera da cirurgia no Hospital de Clínicas da Faculdade de Medicina de Botucatu.

Os pacientes foram avaliados clinicamente no dia anterior à cirurgia, quando foi solicitada ao responsável pela criança a autorização para a inclusão da mesma no estudo, bem como a utilização dos parâmetros clínicos do protocolo da anestesia.

Previamente ao inicio do trabalho foi feito um sorteio para estabelecer a ordem aleatória dos programas farmacocinéticos que seriam utilizados em cada paciente.

Deste modo, os pacientes foram divididos em 2 grupos:

GRUPO S - SHORT (atributos farmacocinéticos de Short ${ }^{14}$ ) - Bolus de propofol de $3 \mathrm{mg} \cdot \mathrm{kg}^{-1}$ e infusão de propofol de $254 \mu \mathrm{g} \cdot \mathrm{kg}^{-1} \cdot \mathrm{min}^{-1}$ (durante 30 minutos), seguida da infusão de propofol de $216 \mu \mathrm{g} \cdot \mathrm{kg}^{-1} \cdot \mathrm{min}^{-1}$ (durante $30 \mathrm{minu}$ tos) - 20 pacientes

GRUPO M - MARSH (atributos farmacocinéticos de Marsh ${ }^{13}$ ) - Bolus de propofol de $3 \mathrm{mg} \cdot \mathrm{kg}^{-1}$ e infusão de propofol de $208 \mu \mathrm{g} \cdot \mathrm{kg}^{-1} \cdot \mathrm{min}^{-1}$ (durante 30 minutos), seguida da infusão de propofol de $170 \mu \mathrm{g} \cdot \mathrm{kg}^{-1} \cdot \mathrm{min}^{-1}$ (durante $30 \mathrm{minu}-$ tos) - 21 pacientes (Tabela I).

Tabela I - Atributos Farmacocinéticos

\begin{tabular}{lll}
\hline & Short $^{14}$ & Marsh $^{13}$ \\
\hline VC $\left(\right.$ L. kg $\left.^{-1}\right)$ & 0,4320 & 0,3430 \\
$\mathrm{~K}_{10}(\min )$ & 0,0635 & 0,0890 \\
$\mathrm{~K}_{12}(\mathrm{~min})$ & 0,2722 & 0,3500 \\
$\mathrm{~K}_{13}(\min )$ & 0,0723 & 0,0770 \\
$\mathrm{~K}_{21}(\min )$ & 0,1023 & 0,1610 \\
$\mathrm{~K}_{31}(\mathrm{~min})$ & 0,0270 & 0,0100 \\
\hline
\end{tabular}


O objetivo destas infusões de propofol em ambos os grupos foi o de atingir a concentração sangüínea alvo de $4 \mu \mathrm{g} \cdot \mathrm{ml}^{-1}$

\section{Técnicas Utilizadas}

Após monitorização e o estabelecimento do acesso venoso, a anestesia foi iniciada com uma dose de alfentanil $30 \mu \mathrm{g} \cdot \mathrm{kg}^{-1}$ seguida do bolus de propofol, $3 \mathrm{mg} \cdot \mathrm{kg}^{-1}$ e de pancurônio 0,08 $\mathrm{mg} . \mathrm{kg}^{-1}$ para se obter relaxamento muscular. Procedeu-se a intubação orotraqueal e instalação da ventilação controlada mecânica, a infusão contínua de propofol e a inalação do $\mathrm{N}_{2} \mathrm{O}$ a $60 \%$. A injeção do bolus e de manutenção de propofol foram feitas através de bomba de infusão. Nos casos em que o paciente apresentou alterações clínicas indicativas de anestesia superficial, foram feitos bolus de alfentanil de $10 \mu \mathrm{g} \cdot \mathrm{kg}^{-1}$. Não foram administradas doses complementares de bloqueador neuromuscular. O último momento estudado foi aos 60 minutos, mesmo quando a cirurgia teve uma duração maior. Programas de computador para calcular as taxas de infusão contínua: programa - Short (PROCHIV) e programa Marsh (PROCRIV).

Ao ser fixado o bolus de $3 \mathrm{mg} \cdot \mathrm{kg}^{-1}$ de propofol para manter concentrações sangüíneas situadas entre 1 a $5 \mu \mathrm{g} \cdot \mathrm{ml}^{-1}$, foram obtidas taxas de infusão apresentadas nas tabelas II e III.

Tabela II - * Concentração Sangüínea Alvo de Propofol $\left(\mu \mathrm{g} \cdot \mathrm{ml}^{-1}\right)^{* *}$ Duração em Minutos das *** Taxas de Infusão $\left(\mu \mathrm{g} \cdot \mathrm{kg}^{-1} \cdot \mathrm{min}^{-1}\right)$ para Manutenção de Anestesia Venosa Contínua, segundo os Atributos Farmacocinéticos de Short ${ }^{14}$. Bolus de $3 \mathrm{mg} \cdot \mathrm{kg}^{-1}$

\begin{tabular}{lccccc}
\hline Minutos & ${ }^{*} 1$ & 2 & 3 & 4 & 5 \\
\hline${ }^{* *} 0-30$ & $* * * 39$ & 110 & 181 & 254 & 324 \\
${ }^{* * 31-90}$ & 52 & 106 & 160 & 216 & 269 \\
\hline
\end{tabular}

Tabela III - *Concentração Sangüínea Alvo de Propofol $\left(\mu \mathrm{g} \cdot \mathrm{ml}^{-1}\right){ }^{* *}$ Duração em Minutos das ${ }^{* * *}$ Taxas de Infusão $\left(\mu \mathrm{g} \cdot \mathrm{kg}^{-1} \cdot \mathrm{min}^{-1}\right)$ para Manutenção de Anestesia Venosa Contínua, segundo os Atributos Farmacocinéticos de Marsh ${ }^{13}$. Bolus de $3 \mathrm{mg} \mathrm{kg}^{-1}$

\begin{tabular}{|c|c|c|c|c|c|}
\hline Minutos & *1 & 2 & 3 & 4 & 5 \\
\hline${ }^{* *} 0-30$ & ***35 & 92 & 150 & 208 & 266 \\
\hline **31- 90 & 38 & 82 & 125 & 170 & 212 \\
\hline
\end{tabular}

Ainjeção venosa de propofol foi realizada através de bomba de infusão cujo princípio obedece à fórmula idealizada por Vianna e Vane ${ }^{15}$, igualando a massa ao volume da droga, ou seja:

$$
\frac{60 *}{C} P D \mathrm{ml} / \text { hora * }
$$

onde:

$\mathrm{C}=$ Concentração da droga $\mu \mathrm{g} \cdot \mathrm{ml}^{-1}$

$\mathrm{P}=$ Peso corporal do paciente $(\mathrm{kg})$

$\mathrm{D}=$ Dose a ser infundida $\mu \mathrm{g}^{-1} \cdot \mathrm{kg}^{-1} \cdot \mathrm{min}^{-1}$

${ }^{*}$ constante (60 minutos $=1$ hora)
Esta bomba facilita a infusão do propofol porque, ao se introduzir a concentração da droga, o peso do paciente e a dose a ser infundida, o equipamento faz automaticamente a conversão da velocidade em $\mathrm{ml} / \mathrm{h}$.

A monitorização foi realizada com eletrocardioscopia, oximetria de pulso $\left(\mathrm{SpO}_{2}\right)$, capnografia $\left(\mathrm{P}_{\mathrm{ET}} \mathrm{CO}_{2}\right)$, medidas continuamente, sendo que a pressão arterial sistólica e diastólica foi avaliada pelo método auscultatório. O controle da profundidade da hipnose foi realizado pelo equipamento de EEG microprocessado denominado BIS.

A ventilação foi controlada mecanicamente com inalação de óxido nitroso (60\%) em oxigênio. A medida da fração inspirada de óxido nitroso $\left(\mathrm{FiN}_{2} \mathrm{O}\right)$ foi avaliada através de capnógrafo.

Para obtenção da concentração sangüínea aproximadamente constante foram idealizados quatro programas de cálculo para propofol: programa-Short (PROCHIV), programa Marsh (PROCRIV), programa-Short(PROPOCHI) e programa - Marsh (PROPOCRI)

Os dois primeiros programas calculavam as taxas de infusão necessárias para manter a concentração no sangue "aproximadamente" constante a partir de um bolus, ou seja, programa - Short (PROCHIV) e programa - Marsh (PROCRIV). Os programas foram iniciados a partir de um comando de execução. Todas as informações são solicitadas automaticamente pelo programa, isto é, nome do paciente, número do registro, idade, peso, sexo, nome do anestesiologista responsável etc. Em seguida, informa-se o bolus inicial e os resultados das taxas de infusão são apresentados numa única tela.

O terceiro (Short-PROPOCHI) e quarto (MarshPROPOCRI) programas calculam as concentrações sangüíneas a partir de um esquema qualquer de administração da droga. Na verdade, estes programas realizam uma simulação ou calculam a concentração predita $(\mathrm{Cp})$ de propofol. Novamente as informações são solicitadas automaticamente. Após a confirmação das informações, é apresentada uma tela com os dados básicos sobre as faixas de dosagens recomendadas. Em seguida, são solicitados, o bolus e a infusão aplicados. O programa aceita qualquer dosagem, mesmo que estas estejam fora das faixas de concentrações típicas. Num pequeno quadro, aparece a concentração calculada a cada $25 \mathrm{~s}$. A qualquer tempo, pode-se promover alterações, ou seja, alterar a infusão e/ou aplicar novo bolus. As informações sempre são solicitadas automaticamente. Não havendo novo bolus ou nova infusão, basta informar o valor "zero" quando solicitado. Este é o caso de quando se encerra a administração da droga e se deseja continuar a simulação para avaliar as condições de recuperação, que não foi objetivo deste trabalho. Finalmente, os resultados são armazenados em arquivos de dados para a visualização das curvas com auxílio de qualquer processador gráfico. Também é fornecida uma listagem dos resultados.

Fundamentalmente, os programas realizam a solução do sistema de equações diferenciais pelo método das diferenças-finitas, também conhecido como método de Euler. A linguagem de programação é o BASIC. 
Dosagem de Propofol Sangüíneo

Aos 20, 40 e 60 minutos (momento 20, momento 40 e momento 60) do início da anestesia foram coletadas amostras de $2 \mathrm{ml}$ de sangue venoso. Estas amostras de sangue foram mantidas a $4^{\circ} \mathrm{C}$ em tubos contendo oxalato de potássio e serviram para a dosagem do propofol sangüíneo $\left(\mathrm{C}_{\mathrm{m}}\right)$.

A dosagem sangüínea de propofol foi feita pelo método da Cromatografia Líquida de Alta Performance (CLAP), usando aparelho Shimadzu Mod. LC 10 com detector fluorométrico (Shimadzu F 535) entre $276 \mathrm{~mm}$ e $310 \mathrm{~mm}$ de comprimento de onda; pressão de 120 psi \pm 20 e fluxo de $1,25 \mathrm{ml} \mathrm{min}^{-1}$, segundo a técnica descrita por Plummer ${ }^{17}$.

Além da medida da concentração sangüínea de propofol $\left(C_{m}\right)$ foi calculada a concentração sangüínea predita de propofol $\left(C_{p}\right)$ através dos programas de computador: programa Short (PROPOCHI) e programa Marsh (PROPOCRI). Com estes atributos foi calculada a Mediana da Performance do Erro (MPE), cujo resultado é obtido em $\pm \%$ através da fórmula:

$$
\text { MPE } \pm \%=C_{m}-C_{p} / C_{p} \times 100
$$

Foi calculada também a Mediana Absoluta da Performance do Erro (MAPE) cujo resultado é semelhante ao MPE; porém, sem o valor negativo ou positivo:

$$
\text { MAPE } \%=C_{m-} C_{p} / C_{p} \times 100
$$

\section{Momentos Estudados}

A coleta dos parâmetros clínicos: índice bispectral (BIS), pressão arterial sistólica (PAS), pressão arterial diastólica (PAD), freqüência cardíaca (FC) e saturação de $\mathrm{O}_{2}$ da hemoglobina $\left(\mathrm{SpO}_{2}\right)$ foi realizada a cada 5 minutos. Para fins de análise estatística, estes atributos foram avaliados nos seguintes momentos:

Momento 0: imediatamente antes da indução anestésica controle $\left(\mathrm{M}_{0}\right)$;

Momento 5: 5 minutos após o início da infusão de propofol $\left(\mathrm{M}_{5}\right)$;

Momento 10: 10 minutos após o início da infusão de propofol - $\left(\mathrm{M}_{10}\right)$;

Momento 20:20 minutos após o início da infusão de propofol - $\left(\mathrm{M}_{20}\right)$;

Momento 40:40 minutos após o início da infusão de propofol - $\left(\mathrm{M}_{40}\right)$;

Momento 60:60 minutos após o início da infusão de propofol - $\left(M_{60}\right)$;

O $\mathrm{FiN}_{2} \mathrm{O}$ e a $\mathrm{P}_{\mathrm{ET}} \mathrm{CO}_{2}$ não foram avaliados no $\mathrm{M}_{0}$.

\section{Métodos Estatísticos}

Para comparação dos grupos de acordo com as variáveis BIS, $\mathrm{FiN}_{2} \mathrm{O}$, $\mathrm{SatO}_{2}$, Mediana Performance do Erro (MPE),
Mediana da Performance do Erro Absoluto (MAPE) e concentração sangüínea de propofol foram utilizados os testes de Friedman para comparação dos momentos dentro de cada grupo. Para comparação dos grupos dentro de cada momento foi utilizado o método de Mann-Whitney. Para as variáveis $\mathrm{PAS}, \mathrm{PAD}, \mathrm{FC}, \mathrm{P}_{\mathrm{ET}} \mathrm{CO}_{2}$ foi utilizada a Análise de Perfil $^{18}$, pois as mesmas têm distribuição normal.

Para verificar a homogeneidade dos grupos para as variáveis idade, altura e peso foi utilizado o teste $t$ de Student e para a variável sexo foi utilizado o teste Exato de Fisher ${ }^{19}$.

No estudo das repetições de bolus de alfentanil dos grupos $S$ e M empregou-se o teste $t$ de Student.

Os resultados foram sempre avaliados ao nível de significância $5 \%$ (Figuras 1 e 2 ).

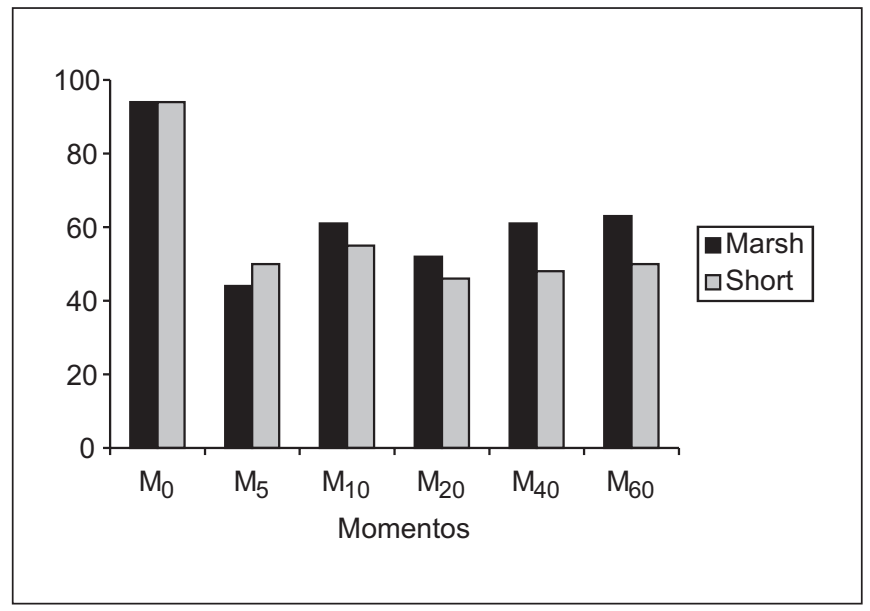

Figura 1 - Valores Mediano do BIS

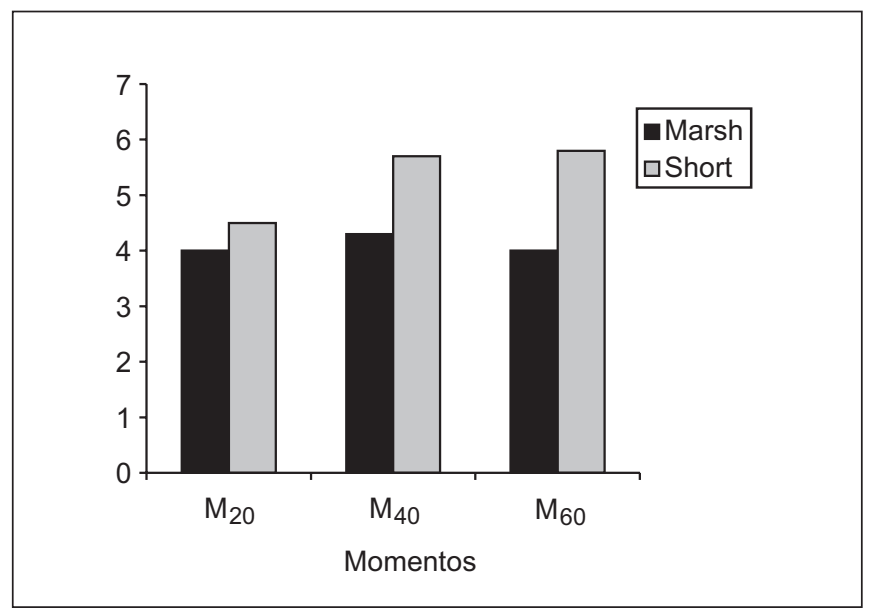

Figura 2 - Valores Medianos da Concentração Sangüínea de Propofol $\left(\mu \mathrm{g} \cdot \mathrm{ml}^{-1}\right)$

\section{RESULTADOS}

Nesta pesquisa houve homogeneidade dos grupos nas variáveis demográficas idade, altura, peso e sexo (Tabelas IV e V). 
MÉTODO SIMPLIFICADO PARA MANUTENÇÃO DA CONCENTRAÇÃO SANGÜÍNEA DE PROPOFOL EM NÍVEL APROXIMADAMENTE CONSTANTE, ASSOCIADO AO ÓXIDO NITROSO NO PACIENTE PEDIÁTRICO

Tabela IV - Média e Desvio Padrão das Variáveis: Idade (anos), Altura (cm) e Peso $(\mathrm{kg})$ segundo Grupos

\begin{tabular}{lccc}
\hline & \multicolumn{3}{c}{ Variável } \\
\hline Grupos & Idade & Altura & Peso \\
\hline Marsh & $6,9 \pm 2,3 \mathrm{a}^{(1)}$ & $122,0 \pm 17,3 \mathrm{a}$ & $25,4 \pm 8,8 \mathrm{a}$ \\
Short & $7,6 \pm 2,5 \mathrm{a}$ & $114,8 \pm 30,4 \mathrm{a}$ & $25,2 \pm 8,3 \mathrm{a}$ \\
Valor de $\mathrm{p}$ & $\mathrm{p}>0,10$ & $\mathrm{p}>0,10$ & $\mathrm{p}>0,10$ \\
\hline
\end{tabular}

(1) médias seguidas de mesma letra não diferem estatisticamente $(p>0,05)$

Tabela V - Distribuição de Freqüências Absolutas e Relativas dos Pacientes segundo Sexo e Grupo

\begin{tabular}{lcc}
\hline & \multicolumn{2}{c}{ Sexo } \\
\hline Grupos & Feminino $(n-\%)$ & Masculino $(\mathrm{n}-\%)$ \\
\hline Marsh & $9(43)$ & $12(57)$ \\
Short & $8(40)$ & $12(60)$ \\
\hline
\end{tabular}

$p>0,05$

Os parâmetros: saturação de $\mathrm{O}_{2}$ da hemoglobina $\left(\mathrm{SpO}_{2}\right)$, concentração inspirada de óxido nitroso $\left(\mathrm{FiN}_{2} \mathrm{O}\right)$, pressão parcial de $\mathrm{CO}_{2}$ ao final da expiração $\left(\mathrm{P}_{\mathrm{ET}} \mathrm{CO}_{2}\right)$, pressão arterial sistólica e diastólica, freqüência cardíaca e de doses repetidas de alfentanil não apresentaram significância entre momentos e entre grupos $(p>0,05)$.

Os resultados que foram estatisticamente significativos são apresentados nas tabelas VI, VII tabela VIII, IX e figuras 3 e 4.

Tabela VI - Valores Medianos do BIS (100-0) segundo Grupos e Momentos

\begin{tabular}{lcccccc}
\hline \multicolumn{7}{c}{ Momentos } \\
\hline Grupos & 0 & 5 & 10 & 30 & 45 & 60 \\
\hline Marsh & $94 \mathrm{~A}^{(1)} \mathrm{a}^{(2)}$ & $44 \mathrm{Ad}$ & $61 \mathrm{Abc}$ & $52 \mathrm{Ab}$ & $61 \mathrm{Ac}$ & $63 \mathrm{Ac}$ \\
Short & $94 \mathrm{Aa}$ & $50 \mathrm{Abc}$ & $55 \mathrm{Ab}$ & $46 \mathrm{Ac}$ & $48 \mathrm{Abc}$ & $50 \mathrm{Abc}$
\end{tabular}

(1) Medianas seguidas de mesma letra maiúscula dentro de cada grupo não diferem estatisticamente $(p>0,05)$

(2) Medianas seguidas de mesma letra minúscula dentro de cada momento não diferem estatisticamente $(p>0,05)$

Tabela VII - Valores Medianos da Concentração Sangüínea de Propofol $\left(\mu \mathrm{g} . \mathrm{ml}^{-1}\right)$ segundo Grupos e Momentos

\begin{tabular}{lccc}
\hline \multicolumn{3}{c}{ Momentos } \\
\hline Grupos & 20 & 40 & 60 \\
\hline Marsh & $4,0 \mathrm{~A}^{(1)} \mathrm{a}^{(2)}$ & $4,3 \mathrm{Aa}$ & $4,0 \mathrm{~B} \mathrm{a}$ \\
Short & $4,5 \mathrm{~A} \mathrm{a}$ & $5,7 \mathrm{Ab}$ & $5,8 \mathrm{~A} \mathrm{a}$ \\
\hline
\end{tabular}

(1) Médias seguidas de mesma letra maiúscula dentro de cada grupo não diferem estatisticamente $(p>0,05)$

(2) Médias seguidas de mesma letra minúscula dentro de cada momento não diferem estatisticamente $(p>0,05)$
Tabela VIII - Valores Medianos da Performance do Erro (MPE) \% segundo Grupos e Momentos

\begin{tabular}{lccc}
\hline \multicolumn{3}{c}{ Momentos } \\
\hline Grupos & 20 & 40 & 60 \\
\hline Marsh & $8,4 \mathrm{~A}^{(1)} \mathrm{a}^{(2)}$ & $16,0 \mathrm{~A} \mathrm{a}$ & $4,6 \mathrm{~B} \mathrm{a}$ \\
Short & $16,1 \mathrm{~A} \mathrm{~b}$ & $12,1 \mathrm{~A} \mathrm{~b}$ & $52,0 \mathrm{~A} \mathrm{a}$ \\
\hline
\end{tabular}

(1) Médias seguidas de mesma letra maiúscula dentro de cada grupo não diferem estatisticamente $(p>0,05)$

(2) Médias seguidas de mesma letra minúscula dentro de cada momento não diferem estatisticamente $(p>0,05)$

Tabela IX - Valores Medianos Absolutos da Performance do Erro (MAPE) \% segundo Grupos e Momentos

\begin{tabular}{lccc}
\hline & \multicolumn{3}{c}{ Momentos } \\
\hline Grupos & 20 & 40 & 60 \\
\hline Marsh & $28,7 \mathrm{~A}^{(1)} \mathrm{a}^{(2)}$ & $33,2 \mathrm{~A} \mathrm{a}$ & $23,6 \mathrm{~B} \mathrm{a}$ \\
Short & $44,7 \mathrm{~A} \mathrm{a}$ & $36,3 \mathrm{~A} \mathrm{a}$ & $53,8 \mathrm{~A} \mathrm{a}$ \\
\hline
\end{tabular}

(1) Médias seguidas de mesma letra maiúscula dentro de cada grupo não diferem estatisticamente $(p>0,05)$

(2) Médias seguidas de mesma letra minúscula dentro de cada momento não diferem estatisticamente $(p>0,05)$

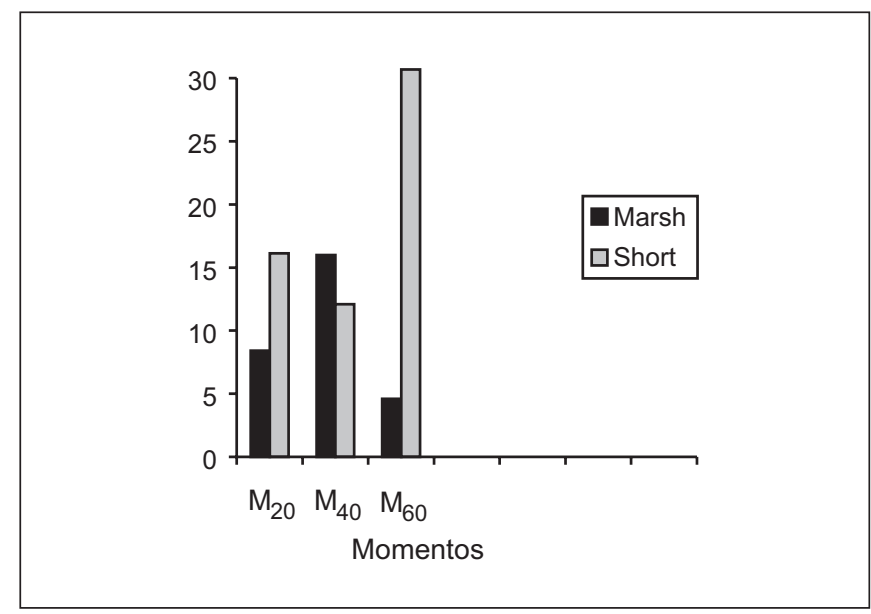

Figura 3 - Valores Medianos do MPE - \%

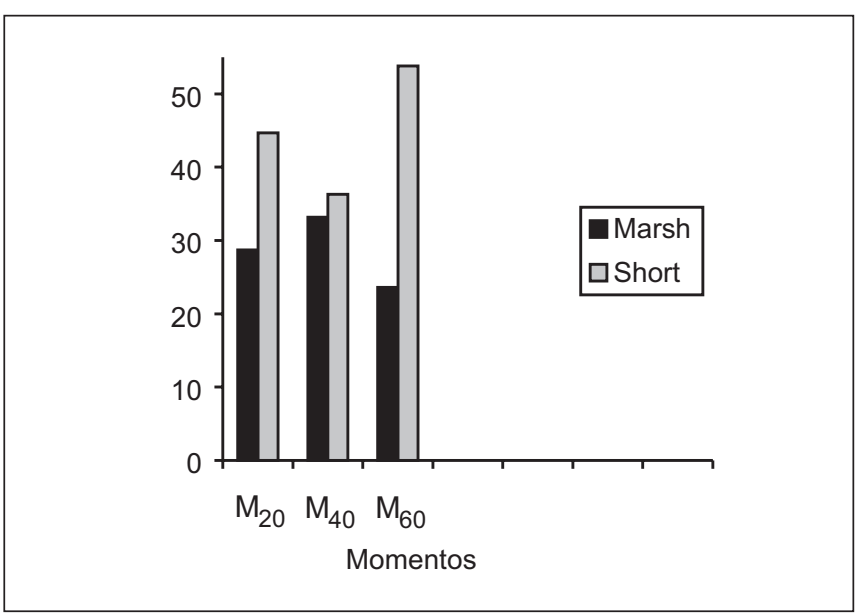

Figura 4 - Valores Medianos do MAPE - \%

Revista Brasileira de Anestesiologia Vol. 52, No 3, Maio - Junho, 2002 


\section{DISCUSSÃO}

$\mathrm{Na}$ atualidade, há um crescente interesse entre anestesiologistas em usar infusão venosa de hipnóticos, analgésicos e bloqueadores neuromusculares. O efeito terapêutico de diversos fármacos é função da concentração na biofase que é determinada pela concentração sangüínea. A concentração sangüínea pode ser mantida por bombas de infusões controladas pelo computador. No nosso meio há um equipamento comercial disponível para uso do propofol denominado Diprifusor ${ }^{\circledR}$. Esta bomba de infusão microprocessada utiliza os atributos farmacocinéticos de pacientes adultos. Desse modo, o uso deste equipamento destina-se exclusivamente a estes indivíduos. Isto porque a farmacocinética da criança é muito diferente da do adulto, principalmente quando se utiliza o propofol ${ }^{20}$. Na criança, as curvas de concentrações do propofol são melhor descritas pelo modelo tricompartimental, com uma meia-vida inicial curta (1,5 a 4,2 minutos), pela ocorrência da redistribuição que é seguida por uma segunda fase (9,3 a 56 minutos) associada à alta depuração metabólica no fígado e em outros locais de grande volume de distribuição. Finalmente, a terceira fase final (209 a 475 minutos) reflete o período de eliminação lenta dos tecidos pouco vascularizados ${ }^{21,22}$. Desse modo, na criança, o volume de distribuição do compartimento central (343 $\mathrm{ml}_{\mathrm{kg}} \mathrm{kg}^{-1}$ ) é maior quando comparado ao do adulto $\left(228 \mathrm{ml}_{\mathrm{kg}} \mathrm{kg}^{-1}\right)$. Isto determinará, no período da indução anestésica, a necessidade de aumentar-se a quantidade de propofol. Nos pacientes adultos, para atingir-se a concentração sangüínea alvo de 4 a $5 \mu \mathrm{g} \cdot \mathrm{ml}^{-1}$, são necessários 1,5 a $2 \mathrm{mg}^{\mathrm{kg}}{ }^{-1}$, enquanto em crianças são necessárias quantidades maiores ${ }^{14}$, ou seja, 3 a $3,5 \mathrm{mg} \cdot \mathrm{kg}^{-1}$. A depuração do propofol também está aumentada em pacientes pediátricos. Marsh ${ }^{13}$ mostrou em crianças uma depuração com valores entre 32 a 57 $\mathrm{ml} . \mathrm{kg}^{-1} \cdot \mathrm{min}^{-1}$. Nos pacientes adultos este parâmetro foi em média de $27 \mathrm{ml} \cdot \mathrm{kg}^{-1} \cdot \mathrm{min}^{-1}$. Então, maiores doses de propofol serão necessárias para produzir e manter níveis sangüíneos compatíveis com o nível de hipnose usado em anestesia. O objetivo de calcular a concentração das drogas preditas no sangue por meio de modelos farmacocinéticos é a obtenção de esquema racional de administração destes fármacos.

As duas principais técnicas para a formulação de modelos farmacocinéticos são: modelo de compartimentos e equações exponenciais. Segundo Glass ${ }^{4}$, esta última é uma simplificação grosseira para a maioria das drogas, enquanto a primeira é mais utilizada, entre outras razões, por oferecer compreensão mais intuitiva do fenômeno farmacocinético. Para a maioria das drogas, este fenômeno pode ser visualizado sob o ponto de vista matemático pelo modelo de três compartimentos. O primeiro compartimento, ou compartimento central, é definido como o compartimento no qual a droga é amostrada, isto é, o sangue. Adroga deixa o compartimento central através de eliminação, principalmente pelos rins e fígado, e é distribuída para outros tecidos ${ }^{23}$.

Entretanto, o trabalho de Bailey ${ }^{9}$ mostra que é possível a obtenção de nível aproximadamente constante da concentra- ção da droga no sangue, utilizando as formas convencionais de infusão. Isto permite ao anestesiologista que não disponha de sistemas de infusão controlados por microprocessador, maioria absoluta no nosso meio, realizar procedimentos muito próximos daqueles feitos automaticamente.

Seguindo a sugestão feita por Kruger-Thiemer ${ }^{1}$ e por Glass ${ }^{4}$, denomina-se este esquema anestésico de BET: um bolus (B) preenche todo o compartimento central em nível desejado de concentração, seguido de uma infusão constante para substituir a droga que está sendo eliminada $(E)$ do compartimento central por excreção ou metabolismo. Superposto a isto, existe uma infusão que declina exponencialmente para substituir a droga sendo transferida $(T)$ para os compartimentos periféricos.

Através das equações do modelo de 3 compartimentos, é muito fácil deduzir que, no regime estacionário, uma infusão contínua variável leva à obtenção de concentração constante no sangue, dada por:

$$
\mathrm{I}=\mathrm{Cpo}-\mathrm{V}_{1} \cdot \mathrm{K}_{10}
$$

Onde:

I = regime de infusão constante

Cpo = concentração desejada

$\mathrm{K}_{10}=$ constante de eliminação

$\mathrm{V}_{1}=$ volume do compartimento central

$$
\begin{aligned}
& {\left[\mu \mathrm{g}^{-1} \cdot \mathrm{kg}^{-1} \cdot \mathrm{min}^{-1}\right]} \\
& {\left[\mu \mathrm{g} \cdot \mathrm{ml}^{-1}\right]} \\
& {\left[\mathrm{min}^{-1}\right]} \\
& {[\mathrm{I}]}
\end{aligned}
$$

Na tentativa de se evitar a queda na curva de concentração $x$ tempo, Bailey ${ }^{9}$ propõe que sejam administrados o bolus inicial e uma infusão contínua por $30 \mathrm{~min}$, em seguida ajusta-se a infusão a cada hora, ou seja, nos intervalos 30-90 min, 90-150 min, 150-210 min e assim por diante.

A rigor, é executado programa de cálculo numérico baseado no método de 3 compartimentos que calcula as taxas de infusão necessárias para que as concentrações atinjam o valor pré-estabelecido nos tempos de 30 e 90 minutos e horas subseqüentes (Tabelas II e III). Em geral, as taxas de infusão são reduzidas em cada etapa, entretanto, dependendo da quantidade do bolus inicial, é possível que haja necessidade de se aumentar a infusão da primeira para a segunda etapa.

Em verdade, o tempo em que é fixado o valor da concentração desejada poderia ser qualquer, e se o tempo das etapas fosse reduzido, a concentração iria se desviar cada vez menos do alvo.

Com a utilização de simulado pelo microcomputador, obtêm-se resultados tão "precisos" quanto aqueles obtidos por Marsh ${ }^{13}$ e Short ${ }^{14}$.

Os resultados obtidos devem ser vistos com alguma crítica, dadas as limitações do próprio modelo de 3 compartimentos. A concentração da droga predita no sangue e obtida por cálculo não é exatamente concordante com a real concentração no sangue do paciente. Isso se deve em primeiro lugar aos valores dos parâmetros farmacocinéticos que são obtidos a partir de medições sobre uma população muito pequena de pacientes. Sob o ponto de vista estatístico, não se pode garantir que estas amostras sejam plenamente representativas do universo. Ainda que os parâmetros sejam normaliza- 
dos pelo peso, pelo sexo e pela idade do paciente, os estados clínicos e as diferenças fisiológicas podem variar ${ }^{24}$. Além disso, mesmo que a concentração da droga no sangue seja exatamente igual àquela calculada, a concentração necessária para a resposta a um dado estímulo é diferente para cada paciente. Por outro lado, esta técnica se justifica porque os eventuais desvios da concentração sangüínea da droga estariam situados dentro dos limites da janela terapêutica da droga. Isto foi o que se observou na presente pesquisa, ou seja, os níveis hemodinâmicos e da profundidade da hipnose permaneceram semelhantes em ambos os grupos estudados. Este estudo mostrou também que as variações individuais existiram e por isto são diferentes dos acurados cálculos realizados pelos computadores.

Outro resultado interessante foi a obtenção, entre grupos, de níveis semelhantes de profundidade da hipnose avaliada pelo BIS, mostrando que as diferentes infusões de propofol calculadas pelos dois programas farmacocinéticos estão situadas na mesma janela terapêutica. Entretanto, deve ser ressaltado que os níveis medianos do BIS foram menores no Grupo $S$ com exceção do $M_{5}$, que foi influenciado pela injeção do bolus inicial do propofol. Estes resultados são originais e desconhece-se na literatura trabalho em pediatria que tenha estudado o BIS acompanhado da dosagem do propofol (Tabela VI e Figura 1). Há apenas estudo que avalia o nível de hipnose em crianças sob o efeito do sevoflurano ${ }^{25,26}$ e sevoflurano associado ao $\mathrm{N}_{2} \mathrm{O}^{27}$

Não existe método capaz de avaliar a profundidade da analgesia entre grupos. O único dado para demonstrar que os grupos, neste parâmetro, foram semelhantes, foi a inexistência de significância da complementação da anestesia através de bolus de alfentanil. Esta complementação foi mínima e se resumiu a quatro pacientes do grupo $S$ e a 5 do grupo M. Isto demonstra a eficácia da analgesia do $\mathrm{N}_{2} \mathrm{O}$ a $60 \%$ associado ao propofol.

Neste estudo, utilizou-se, de modo original, atributos farmacocinéticos obtidos de duas pesquisas realizadas em crianças nas faixas etárias de 4 a 12 anos ${ }^{13,14}$. Para uso destes parâmetros farmacocinéticos empregou-se "software" (PROCRIV, PROCHIV) baseado no estudo realizado por Bailey ${ }^{9}$ propondo método simplificado para manter a concentração sangüínea em níveis aproximadamente constantes. A técnica foi criada para capacitar o anestesiologista a manter aproximadamente constante uma concentração sangüínea-alvo desejada. Após dose bolus arbitrária, calculam-se as taxas de infusões decrescentes para manter nível sangüíneo estável. Estas taxas de infusões são obtidas através de equações que calculam o esquema das taxas de infusões seqüenciais. Aacurácia desta técnica foi calculada pela Mediana da Performance do Erro (MPE). É consenso mundial considerar aceitáveis para uso clínico os programas que utilizam atributos de farmacocinética cujo MPE é igual ou menor que 30 . Os resultados da avaliação do MPE desta pesquisa mostraram que os níveis medianos foram 8,4 no momento 20 do grupo Marsh. Depois deste período, o MPE elevou-se para 16 no momento 40 e reduzindo-se para 4,6 no momento
60, mostrando que após uma hora a concentração predita aproxima-se da medida. O programa baseado nos atributos de Short ${ }^{14}$ teve um desempenho inverso, ou seja, o MPE foi de 16 aos 20 minutos de infusão e 12,1 e 52 aos 40 e 60 minutos de infusão de propofol (Tabela VII e Figura 4). Os resultados desta pesquisa mostraram que os atributos farmacocinéticos de Marsh ${ }^{13}$ tiveram uma boa performance e se situaram em limites inferiores ao \pm 30 . Quando os resultados das concentrações preditas e medidas foram avaliados, através da Mediana Absoluto Performance do Erro (MAPE), obtiveram-se os valores absolutos de 28,7 para o momento 20 , 33,2 para o momento 40 e 23,6 para o momento 60 , no grupo $\mathrm{M}$; no grupo $S$ estes valores foram de 44,7 no momento 20 , 36,3 no momento 40 e 53,8 no momento 60 . Estes resultados mostram que durante uma hora de infusão de propofol há uniformidade de resultados nos atributos farmacocinéticos de Marsh ${ }^{13}$, enquanto na infusão programada para o grupo $S$ existe um efeito, cumulativo deste hipnótico e por isto no momento 60 houve, também, diferença estatisticamente significativa entre ambos os grupos.

O principal objetivo desta pesquisa foi verificar se a escolha do parâmetro farmacocinético de propofol associado ao $\mathrm{N}_{2} \mathrm{O}$ para criança era capaz de influenciar significativamente o nível da hipnose e da anestesia (Tabela VI e Figura 1) Em ambos os grupos não houve diferenças significativas nos parâmetros hemodinâmicos (pressão arterial sistólica e diastólica e a freqüência cardíaca). $\mathrm{ASpO}_{2}$, a concentração inspirada de óxido nitroso $\left(\mathrm{FiN}_{2} \mathrm{O}\right)$ e a $\mathrm{P}_{\mathrm{ET}} \mathrm{CO}_{2}$ também não apresentaram significância entre momentos e entre grupos. Do mesmo modo, a quantidade de analgésico complementar foi similar entre os grupos. Isto demonstra serem os programas farmacocinéticos similares do ponto de vista clínico. Este resultado é compreensível porque mesmo existindo diferença do ponto de vista farmacocinético, as concentrações sangüíneas alvo associadas ao $\mathrm{N}_{2} \mathrm{O}$ permaneceram dentro da janela terapêutica. Neste estudo os programas farmacocinéticos foram obtidos de pacientes pediátricos originários de dois continentes (Europa e Ásia) e aplicados a crianças do Brasil. O mais correto é acreditar que o programa farmacocinético de Short ${ }^{14}$ e originário de crianças de Hong Kong superestimaram a concentração sangüínea alvo de pacientes pediátricos brasileiros. De modo oposto, o programa farmacocinético de Marsh ${ }^{13}$, obtidos de crianças européias, mostrou-se satisfatório para obtenção da concentração-alvo de propofol de $4 \mu \mathrm{g} \cdot \mathrm{ml}^{-1}$ em nossos pacientes.

Do ponto de vista clínico, os pacientes tiveram comportamentos semelhantes usando técnica mais precisa de avaliação de hipnose, como é o caso do uso do equipamento de EEG microprocessado denominado BIS (Tabela VI e Figura 1). Já do ponto de vista laboratorial, obtida pela medida da concentração sangüínea de propofol, esta similaridade não existiu (Tabelas VII, VIII, IX e Figuras 2, 3 e 4).

Concluindo, a comparação entre os atributos obtidos por Marsh ${ }^{13}$ e Short ${ }^{14}$ mostrou que ambos os programas de computador podem ser usados em pediatria, mas a nossa preferência recai sobre o programa PROCHIV-Marsh por utilizar

Revista Brasileira de Anestesiologia Vol. 52, No 3, Maio - Junho, 2002 
menor infusão de propofol associado ao $\mathrm{N}_{2} \mathrm{O}$, atingir a concentração-alvo com mais acurácia e obter níveis adequados de hipnose em crianças, na faixa etária entre 4 e 12 anos.

\section{Simplified Method to Maintain Propofol Blood Concentration in an Approximately Constant Level Associated to Nitrous Oxide in Pediatric Patients}

Pedro Thadeu Galvão Vianna, TSA, M.D., Elizabeth Pricoli Vilela, M.D., Francisco Carlos Obata Cordon, M.D., Lídia Raquel de Carvalho M.D.

\section{INTRODUCTION}

Kruger-Thiemer ${ }^{1}$ has equated a way of infusing a drug as a function of time, aiming at reaching and maintaining a constant concentration in the central compartment, provided its pharmacokinetics could be described by a linear multicompartmental model ${ }^{2,3}$. This method, developed in clinical practice for continuous intravenous infusion, had a growing interest in the $70 \mathrm{~s}$, though its commercial introduction came more recently, in the $90 \mathrm{~s}^{4}$.

Drug release is defined as automatic when a mechanical or electronic device adjusts the doses, requiring no human intervention (manual). Microcomputers, increasingly accepted in medicine, have allowed this automatic drug release. So, the pharmacokinetic model of a certain anesthetic drug, processed by a computer program, allows its administration according to the desired plasma concentration ${ }^{5}$. Similarly, it is possible to determine when, after the anesthetic infusion withdrawing, the concentration compatible with consciousness recovery will be reached.

The success of this anesthetic technique is largely dependent on reducing operational costs. This type of infusion requires a microprocessor controlled pump like $\mathrm{CACl}$ - Computerized Assisted Continuous Infusion system ${ }^{4,6-8}$, which is not available for most anesthetic procedures.

In 1993, Bailey ${ }^{9}$ developed an alternative method for such infusion, which constantly varies between two or more fixed intravenous continuous infusion rates, which decrease in a controlled manner. Ultimately, he theoretically described a technique to calculate the rhythms of sequential infusions needed to get close to the constant blood level desired.

This method was adapted by Vianna ${ }^{10-12}$ for propofol continuous infusion ${ }^{11}$, using Marsh ${ }^{13}$ and Short ${ }^{14}$ pharmacokinetic parameters with the purpose of spreading the use of target-controlled concentration, that is, the maintenance of an approximately constant desirable blood concentration.

The aim of this study was compare, in clinical and laboratorial terms, Marsh's ${ }^{13}$ and Short's ${ }^{14}$ pharmacokinetic parameters used for target-controlled propofol infusion, associated to nitrous oxide, in children aged 4 to 12 years.

\section{METHODS}

After the Faculdade de Medicina de Botucatu Ethical Committee's, approval 41 pediatric patients of both genders, aged 4 to 12 years, physical status ASA I, scheduled for adenotonsillectomies, strabismus corrections and bone fractures open reductions under general anesthesia were included in the study. Patients were admitted to Hospital das Clinicas, Faculdade de Medicina de Bauru the day before surgery.

After admission, patients were clinically evaluated and parents or guardians were asked to authorize their inclusion on the study, as well as the use of clinical parameters of the anesthetic protocol.

Patients were randomly allocated into two groups, according to the pharmacokinetic program to be used:

GROUP S - SHORT (Short's ${ }^{14}$ pharmacokinetic parameters) - $3 \mathrm{mg} \cdot \mathrm{kg}^{-1}$ bolus propofol and $254 \mu \mathrm{g} \cdot \mathrm{kg}^{-1} \cdot \mathrm{min}^{-1}$ propofol infusion for 30 minutes, followed by 216 $\mu \mathrm{g} \cdot \mathrm{kg}^{-1} \cdot \mathrm{min}^{-1}$ propofol infusion for 30 minutes -20 patients.

GROUP M - MARSH (Marsh's ${ }^{13}$ pharmacokinetic parameters) $-3 \mathrm{mg} \cdot \mathrm{kg}^{-1}$ bolus propofol and $208 \mu \mathrm{g} \cdot \mathrm{kg}^{-1} \cdot \mathrm{min}^{-1}$ for 30 minutes, followed by $170 \mu \mathrm{g} \cdot \mathrm{kg}^{-1} \cdot \mathrm{min}^{-1}$ for 30 minutes - 21 patients (Table I).

The goal of propofol infusions in both groups was to obtain target $4 \mu \mathrm{g} \cdot \mathrm{ml}^{-1}$ blood concentration.

Table I - Pharmacokinetic Parameters

\begin{tabular}{lll}
\hline & Short $^{14}$ & Marsh $^{13}$ \\
\hline $\mathrm{VC}\left(\mathrm{L} . \mathrm{kg}^{-1}\right)$ & 0.4320 & 0.3430 \\
$\mathrm{~K}_{10}(\mathrm{~min})$ & 0.0635 & 0.0890 \\
$\mathrm{~K}_{12}(\mathrm{~min})$ & 0.2722 & 0.3500 \\
$\mathrm{~K}_{13}(\mathrm{~min})$ & 0.0723 & 0.0770 \\
$\mathrm{~K}_{21}(\mathrm{~min})$ & 0.1023 & 0.1610 \\
$\mathrm{~K}_{31}(\mathrm{~min})$ & 0.0270 & 0.0100 \\
\hline
\end{tabular}

Anesthetic Techniques

After monitoring and venous accessing, anesthesia was induced with $30 \mu \mathrm{g} \cdot \mathrm{kg}^{-1}$ alfentanil followed by $3 \mathrm{mg} \cdot \mathrm{kg}^{-1}$ bolus propofol and $0.08 \mathrm{mg} \cdot \mathrm{kg}^{-1}$ pancuronium for muscle relaxation. Next, patients were intubated and controlled mechanical ventilation was started with $60 \% \mathrm{~N}_{2} \mathrm{O} / \mathrm{O}_{2}$, as well as propofol continuous infusion. Propofol bolus and maintenance infusion were administered through an infusion pump. Should the patient exhibit clinical changes indicating superficial anesthesia, $10 \mu \mathrm{g} \cdot \mathrm{kg}^{-1}$ bolus alfentanil were given. No additional neuromuscular blocker doses were given. The last moment studied was at 60 minutes, even when surgery lasted longer. 
Computer programs to calculate continuous infusion rates were: Short (PROCHIV) program and March (PROCRIV) program.

Tables II and III show infusion rates obtained after fixing the 3 $\mathrm{mg} \cdot \mathrm{kg}^{-1}$ bolus propofol to achieve blood concentrations between 1 and $5 \mu \mathrm{g} \cdot \mathrm{ml}^{-1}$.

Table II - * Propofol Target Blood Concentration ( $\mu \mathrm{g} \cdot \mathrm{ml}^{-1}$ ) ${ }^{* * *}$ Infusion Rates $\left(\mu \mathrm{g} \cdot \mathrm{kg}^{-1} \cdot \mathrm{min}^{-1}\right){ }^{* *}$ Duration in Minutes to Maintain Continuous Intravenous Anesthesia according to Short's ${ }^{14}$ Pharmacokinetic Parameters. $3 \mathrm{mg} . \mathrm{kg}^{-1}$ bolus

\begin{tabular}{lccccc}
\hline Minutes & ${ }^{*} 1$ & 2 & 3 & 4 & 5 \\
\hline${ }^{* *} 0-30$ & ${ }^{* * * 39}$ & 110 & 181 & 254 & 324 \\
${ }^{* *} 31-90$ & 52 & 106 & 160 & 216 & 269 \\
\hline
\end{tabular}

Table III - ** Propofol Target Blood Concentration $\left(\mu \mathrm{g} \cdot \mathrm{ml}^{-1}\right)$ ***Infusion Rates $\left(\mu \mathrm{g} . \mathrm{kg}^{-1} \cdot \mathrm{min}^{-1}\right){ }^{* *}$ Duration in Minutes to Maintain Continuous Intravenous Anesthesia according to Marsh's ${ }^{13}$ Pharmacokinetic Parameters. $3 \mathrm{mg} \cdot \mathrm{kg}^{-1}$ bolus

\begin{tabular}{|c|c|c|c|c|c|}
\hline Minutes & $* 1$ & 2 & 3 & 4 & 5 \\
\hline **0-30 & ***35 & 92 & 150 & 208 & 266 \\
\hline${ }^{* *} 31-90$ & 38 & 82 & 125 & 170 & 212 \\
\hline
\end{tabular}

Intravenous propofol infusion was made through an infusion pump according to the formula developed by Vianna and Vane ${ }^{15}$, equaling mass to drug volume, which is:

$$
\frac{60^{*}}{C} W D \mathrm{ml} / \text { hour * }
$$

where:

$\mathrm{C}=$ drug concentration $\mathrm{I}\left(\mu \mathrm{g} \cdot \mathrm{ml}^{-1}\right)$

$\mathrm{W}=$ patient's body weight $(\mathrm{kg})$

$\mathrm{D}=$ infusion rate $\left(\mu \mathrm{g} \cdot \mathrm{kg}^{-1} \cdot \mathrm{min}^{-1}\right)$

${ }^{*}$ constant $(60$ minutes $=1$ hour $)$

This pump makes easy propofol infusion, since as you enter drug's concentration, patient's weight and dose to be infused, the equipment automatically calculates the infusion rate in $\mathrm{ml}^{-\mathrm{h}^{-1}}$.

Monitoring consisted of ECG, pulse oximetry $\left(\mathrm{SpO}_{2}\right)$, capnography $\left(\mathrm{P}_{\mathrm{ET}} \mathrm{CO}_{2}\right)$ and blood pressure measured by auscultatory method. Hypnosis depth was assessed by a microprocessed EEG equipment (BIS).

Ventilation was mechanically controlled, with nitrous oxide inhalation $(60 \%)$ in oxygen. Nitrous oxide inspired fraction $\left(\mathrm{FiN}_{2} \mathrm{O}\right)$ was evaluated a gas analyzer.

Four programs were developed to obtain approximately constant propofol blood concentration: Short (PROCHIV) program, Marsh (PROCRIV) program, Short (PROPOCHI) program and Marsh (PROPOCRI) program.

The first two programs Short (PROCHIV) and Marsh (PROCRIV) calculated propofol infusion rates needed to maintain "almost" constant blood concentration after a given bolus.

Starting by an execution command, the programs prompt for patient's name, record number, age, weight, gender, anesthesiologist's name, etc. Following, the initial propofol bolus was informed and the programs return the infusion rates to be used in a single screen.

The third (Short - PROPOCHI) and fourth (Marsh PROPOCRI) programs calculate blood concentrations after any drug administration scheme. In fact, these programs either emulate or calculate propofol predicted concentration $(P c)$. Again, patient's information is automatically asked. After confirmation, a screen is displayed with basic data about recommended dose ranges. Then, applied bolus and infusion rate are asked. The program accepts any dosage, even outside typical concentration ranges. A small window shows calculated concentration at every $25 \mathrm{~s}$. New bolus or changes in infusion rate can be made at any time. The programs are always prompting for new information. If there is no new bolus or new infusion, one just inform value "zero" when asked. This is the case when the drug is no longer being administered, but emulation should continue to evaluate recovery conditions, which was not the objective of our study. Finally, results are stored in data files for curves visualization through any graphic processor. A list of results is also supplied.

Basically, programs solve the differential equations system by the finite differences method, also known as Euler's method. Programming language is BASIC.

\section{Blood Propofol Measurement}

At 20, 40 and 60 minutes (moment 20, moment 40 and moment 60 ) after beginning of anesthesia, $2 \mathrm{ml}$ of venous blood samples were collected. These samples were maintained at $4{ }^{\circ} \mathrm{C}$ in tubes with potassium oxalate and were used to determine blood propofol concentration $\left(C_{m}\right)$. Blood propofol was measured by High Performance Liquid Chromoatography (HPLC) using a Shimadzu Mod. LC 10 device with fluorometric detector (Shimadzu F 535), with wavelength between $276 \mathrm{~mm}$ and $310 \mathrm{~mm}$, pressure of 120 psi \pm 20 and flow of $1.25 \mathrm{ml} \mathrm{min}^{-1}$, according to Plummer's ${ }^{17}$ technique.

Measured propofol blood concentration $\left(\mathrm{C}_{\mathrm{m}}\right)$ and propofol predicted concentration $\left(C_{p}\right)$ by Short program (PROPOCHI) and Marsh program (PROPOCRI) were then statistically analyzed. Error Performance Median (EPM) was derived, in $\pm \%$, through following the formula:

$$
E P M \pm \%-C_{m}-C_{p} / C_{p} \times 100
$$

Error Performance Absolute Median (EPAM) was also calculated, which result is similar to EPM, however without positive or negative value:

$$
\text { EPAM \% }=C_{m}-C_{p} / C_{p} \times 100
$$




\section{Studied Moments}

Clinical parameters like bispectral index (BIS), systolic blood pressure (SBP), diastolic blood pressure (DBP), heart rate $(\mathrm{HR})$ and hemoglobin oxygen saturation $\left(\mathrm{SpO}_{2}\right)$ were registered every 5 minutes. For statistical analysis, those attributes were evaluated in the following moments:

Moment 0 - immediately before anesthetic induction - control $\left(\mathrm{M}_{0}\right)$;

Moment 5 - 5 minutes after beginning of propofol infusion $\left(\mathrm{M}_{5}\right)$;

Moment 10 - 10 minutes after beginning of propofol infusion - $\left(\mathrm{M}_{10}\right)$

Moment 20 - 20 minutes after beginning of propofol infusion $-\left(\mathrm{M}_{20}\right)$

Moment 40 - 40 minutes after beginning of propofol infusion - $\left(\mathrm{M}_{40}\right)$

Moment 60 - 60 minutes after beginning of propofol infusion - $\left(\mathrm{M}_{60}\right)$;

$\mathrm{FiN}_{2} \mathrm{O}$ and $\mathrm{P}_{\mathrm{ET}} \mathrm{CO}_{2}$ were not evaluated in $\mathrm{M}_{0}$.

\section{Statistical Methods}

Friedman's tests were used to compare BIS, $\mathrm{FiN}_{2} \mathrm{O}, \mathrm{SatO}_{2}$, Error Performance Median (EPM), Error Performance Absolute Median (EPAM) and propofol blood concentration between moments within each group. Mann-Whitney test was used to compare groups in each moment. Profile Analysis ${ }^{18}$ was used for SBP, DBP, HR and $\mathrm{P}_{\mathrm{ET}} \mathrm{CO}_{2}$, because they present normal distribution.

Student's $t$ test was applied to check groups homogeneity regarding to age, height and weight. Fisher's Exact test was used for gender ${ }^{19}$.

Student's $t$ test was used to compare alfentanil bolus repetition between groups $S$ and $M$.

Results were always evaluated at a significance level established to $5 \%$ (Figures 1 and 2).

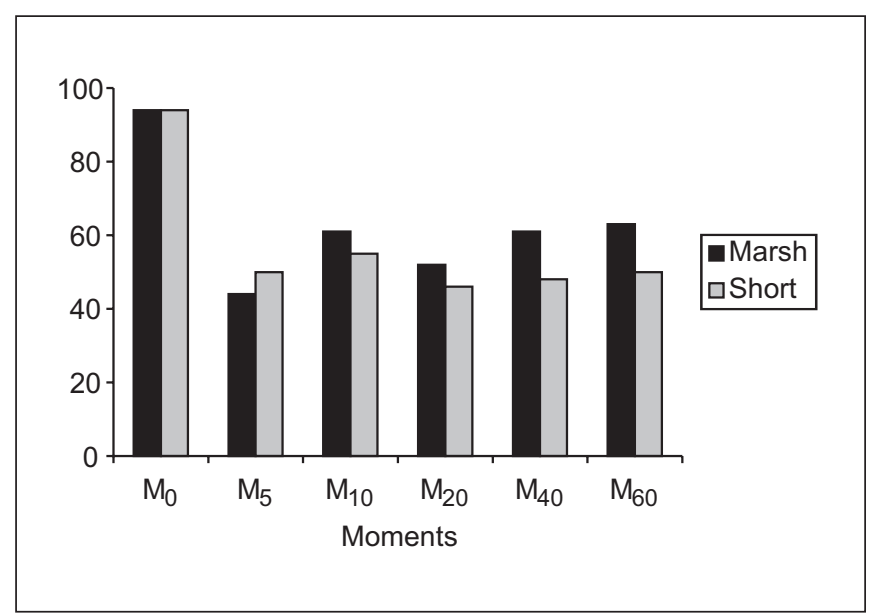

Figure 1 - Median BIS Values

Revista Brasileira de Anestesiologia

Vol. 52, № 3, Maio - Junho, 2002

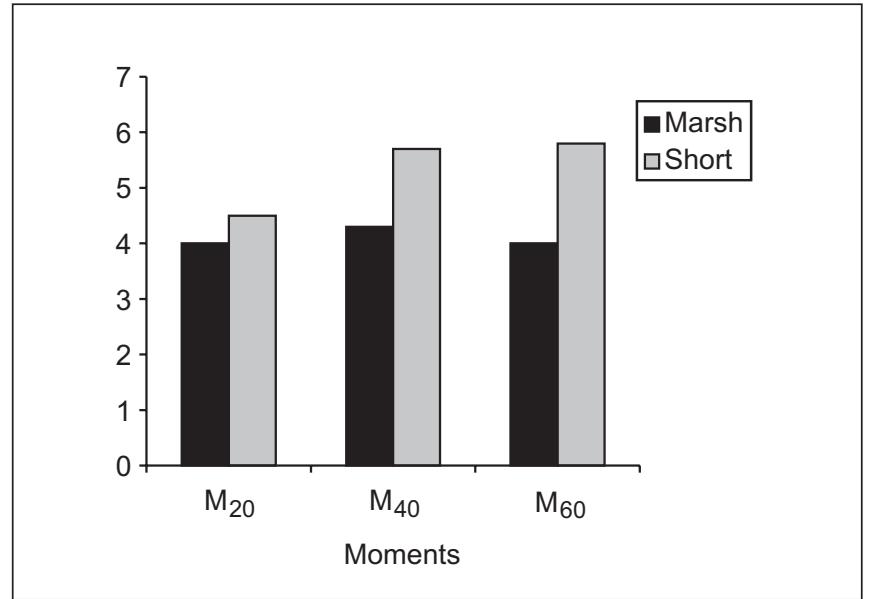

Figure 2 - Mean Propofol Blood Concentration Values $\left(\mu \mathrm{g} \cdot \mathrm{ml}^{-1}\right)$

\section{RESULTS}

Groups were homogeneous in age, height, weight and gender (Tables IV and V).

Table IV - Age (years), Height (cm) and Weight (kg) Mean and Standard Deviation according to Groups

\begin{tabular}{lccc}
\hline & \multicolumn{3}{c}{ Variable } \\
\hline Groups & Age & Height & Weight \\
\hline Marsh & $6.9 \pm 2.3 \mathrm{a}^{(1)}$ & $122.0 \pm 17.3 \mathrm{a}$ & $25.4 \pm 8.8 \mathrm{a}$ \\
Short & $7.6 \pm 2.5 \mathrm{a}$ & $114.8 \pm 30.4 \mathrm{a}$ & $25.2 \pm 8.3 \mathrm{a}$ \\
$\mathrm{p}$ value & $\mathrm{p}>0.10$ & $\mathrm{p}>0.10$ & $\mathrm{p}>0.10$ \\
\hline
\end{tabular}

(1) means followed by the same letter are not statistically different $(p>0.05)$

Table V - Patients Absolute and Relative Frequency According to Gender and Group

\begin{tabular}{lcc}
\hline \multicolumn{3}{c}{ Gender } \\
\hline Groups & Female $(n-\%)$ & Male $(n-\%)$ \\
\hline Marsh & $9(43)$ & $12(57)$ \\
Short & $8(40)$ & $12(60)$ \\
\hline$p>0.05$ & &
\end{tabular}

There were no significant differences between moments and between groups in hemoglobin oxygen saturation $\left(\mathrm{SpO}_{2}\right)$, nitrous oxide inspired concentration $\left(\mathrm{FiN}_{2} \mathrm{O}\right)$, end tidal $\mathrm{CO}_{2}$ partial pressure $\left(\mathrm{P}_{\mathrm{ET}} \mathrm{CO}_{2}\right)$, systolic and diastolic blood pressure, heart rate and repeated alfentanil doses ( $p$ $>0.05$ ).

Statistically significant results are shown in tables VI, VII, VIII, IX and figures 3 and 4. 
Table VI - Median BIS Values (100-0) According to Groups and Moments

\begin{tabular}{lcccccc}
\hline \multicolumn{6}{c}{ Moments } \\
\hline Groups & 0 & 5 & 10 & 30 & 45 & 60 \\
\hline Marsh & $94 \mathrm{~A}^{(1)} \mathrm{a}^{(2)}$ & $44 \mathrm{Ad}$ & $61 \mathrm{Abc}$ & $52 \mathrm{Ab}$ & $61 \mathrm{Ac}$ & $63 \mathrm{Ac}$ \\
Short & $94 \mathrm{~A} \mathrm{a}$ & $50 \mathrm{Abc}$ & $55 \mathrm{Ab}$ & $46 \mathrm{Ac}$ & $48 \mathrm{Abc}$ & $50 \mathrm{Abc}$ \\
\hline
\end{tabular}

(1) Medians followed by the same upper case letter within each group are not statistically different $(p>0.05)$

(2) Medians followed by the same lower case letter within each moment are not statistically different $(p>0.05)$

Table VII - Median Propofol Blood Concentration Values $\left(\mu \mathrm{g} \cdot \mathrm{ml}^{-1}\right)$ according to Groups and Moments

\begin{tabular}{lccc}
\hline & \multicolumn{3}{c}{ Moments } \\
\hline Groups & 20 & 40 & 60 \\
\hline Marsh & $4.0 \mathrm{~A}^{(1)} \mathrm{a}^{(2)}$ & $4.3 \mathrm{~A} \mathrm{a}$ & $4.0 \mathrm{~B} \mathrm{a}$ \\
Short & $4.5 \mathrm{~A} \mathrm{a}$ & $5.7 \mathrm{~A} \mathrm{~b}$ & $5.8 \mathrm{~A} \mathrm{a}$ \\
\hline
\end{tabular}

(1) Medians followed by the same upper case letter within each group are not statistically different $(p>0.05)$

(2) Medians followed by the same lower case letter within each moment are not statistically different $(p>0.05)$

Table VIII - Median Error Performance (MEP) Values (\%) according to Groups and Moments

\begin{tabular}{lccc}
\hline \multicolumn{4}{c}{ Moments } \\
\hline Groups & 20 & 40 & 60 \\
\hline Marsh & $8.4 \mathrm{~A}^{(1)} \mathrm{a}^{(2)}$ & $16.0 \mathrm{~A} \mathrm{a}$ & $4.6 \mathrm{~B} \mathrm{a}$ \\
Short & $16.1 \mathrm{Ab}$ & $12.1 \mathrm{Ab}$ & $52.0 \mathrm{~A} \mathrm{a}$ \\
\hline
\end{tabular}

(1) Medians followed by the same upper case letter within each group are not statistically different $(p>0.05)$

(2) Medians followed by the same lower case letter within each moment are not statistically different $(p>0.05)$

Table IX - Medians Absolute Performance Error (MAPE) values (\%) according to Groups and Moments

\begin{tabular}{lccc}
\hline & \multicolumn{3}{c}{ Moments } \\
\hline Groups & 20 & 40 & 60 \\
\hline Marsh & $28.7 \mathrm{~A}^{(1)} \mathrm{a}^{(2)}$ & $33.2 \mathrm{~A} \mathrm{a}$ & $23.6 \mathrm{~B} \mathrm{a}$ \\
Short & $44.7 \mathrm{~A} \mathrm{a}$ & $36.3 \mathrm{~A} \mathrm{a}$ & $53.8 \mathrm{~A} \mathrm{a}$ \\
\hline
\end{tabular}

(1) Medians followed by the same upper case letter within each group are not statistically different $(p>0.05)$

(2) Medians followed by the same lower case letter within each moment are not statistically different $(p>0.05)$

\section{DISCUSSION}

There is currently an increasing interest of anesthesiologists in using intravenous hypnotics, analgesics and neuromuscular blockers intravenous continuous infusions. The therapeutic effect of different drugs is a function of their concentration in the biophase, which is determined by blood concentration. Blood concentration may be maintained by computer assisted infusion pumps. There is a commercially available equipment in Brazil for propofol infusion, called Diprifusor $^{\circledR}$. This microprocessed infusion pump uses

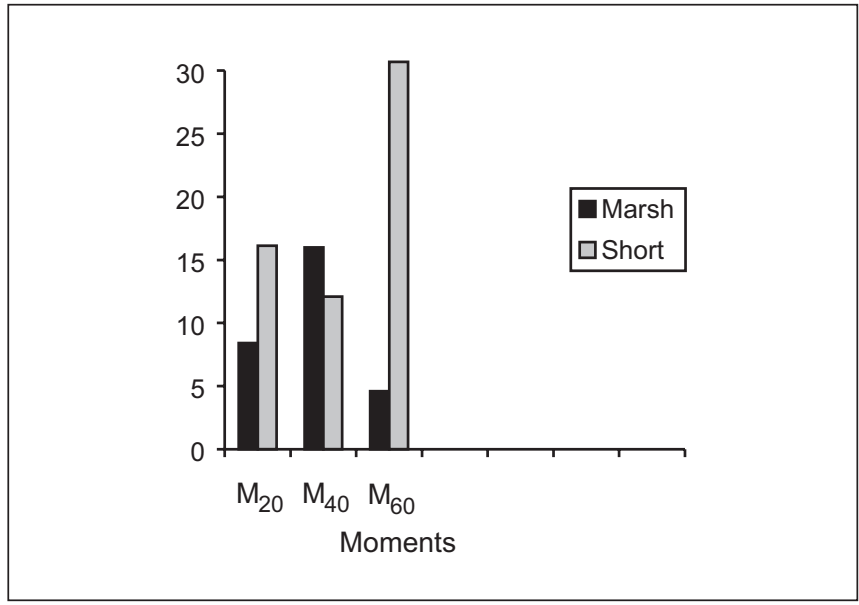

Figure 3 - Mean EPM Values - \%

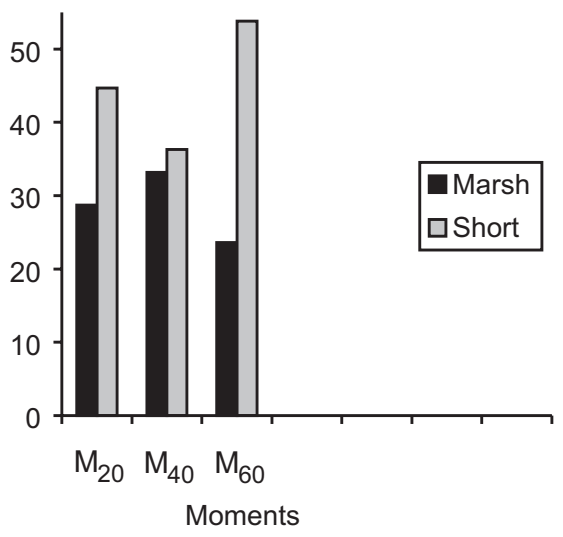

Figure 4 - Mean EPAM values - \%

pharmacokinetic parameters of adult patients, therefore it and is exclusive for them since children's pharmacokinetics is very different from adults, especially when propofol is used ${ }^{20}$. Propofol concentration curves in children are better described by a tricomparmental model, with a short initial half-life (1.5 to 4.2 minutes), due to redistribution process, followed by a second phase ( 9.3 to 56 minutes) associated to high metabolic clearance in the liver and in other large distribution volume sites. The third and final phase (209 to 475 minutes) reflects the slow elimination process of less vascularized tissues ${ }^{21,22}$. So, in children, central compartment distribution volume $\left(343 \mathrm{ml} . \mathrm{kg}^{-1}\right)$ is higher as compared to adults $\left(228 \mathrm{ml} . \mathrm{kg}^{-1}\right)$. This determines the need to increase propofol doses during anesthetic induction. In adult patients, to reach a blood target-concentration of 4 to $5 \mu \mathrm{g} \cdot \mathrm{ml}^{-1}, 1.5$ to 2 $\mathrm{mg} . \mathrm{kg}^{-1}$ venous propofol are needed, while in children require higher doses ${ }^{14}$, like 3 to $3.5 \mathrm{mg} \mathrm{kg}^{-1}$. Propofol clearance is also increased in pediatric patients. Marsh ${ }^{13}$ has shown in children propofol clearances of 32 to 57 $\mathrm{ml} . \mathrm{kg}^{-1} \cdot \mathrm{min}^{-1}$. In adult patients, this parameter was, in average, $27 \mathrm{ml} . \mathrm{kg}^{-1} \cdot \mathrm{min}^{-1}$. So, higher propofol doses are required

Revista Brasileira de Anestesiologia Vol. 52, No 3, Maio - Junho, 2002 
to achieve and maintain blood levels compatible with the hypnosis needed in anesthesia.

The aim of calculating predicted drugs blood concentrations by pharmacokinetic models is to obtain a rational regimen for those drugs administration.

The two major pharmacokinetic model techniques are: compartment model and exponential equations. According to Glass ${ }^{4}$, the latter is a rough simplification for most drugs, while the former is more widely used for offering an intuitive understanding of the pharmacokinetic phenomenum, among other reasons.

For most drugs, this phenomenum can be mathematically reproducted by the three compartments model. The first, or central compartment, is defined as the compartment where the drugs can be sampled, that is, the blood. Drugs leave the central compartment by elimination, especially by kidneys and by distribution to other tissues as well ${ }^{23}$.

However, Bailey ${ }^{9}$ has shown that it is possible to obtain approximately constant drug blood concentrations using conventional infusion techniques.

This allows anesthesiologist's working in places where computer assisted infusion systems are not available, like the majority in Brazil, to perform procedures very close to those automatically performed.

According to a suggestion of Kruger-Thiemer ${ }^{1}$ and Glass ${ }^{4}$, this anesthetic infusion regimen is called BET: a bolus (B) fills the whole central compartment reaching the desired drug concentration, followed by a constant infusion to replace the drug being eliminated ( $E$ ) from this compartment by excretion or metabolism. Superimposed to it, another infusion, which exponentially decreases with time, is used to replace the drug being transferred $(T)$ to peripheral compartments. Through the three compartments model equations, it is very easy to assume that, in the steady state, a variable continuous infusion rate leads to a constant blood concentration given by:

$$
\mathrm{I}=\mathrm{Cpo}-\mathrm{V}_{1} \cdot \mathrm{K}_{10}
$$

Where:

I = constant infusion regimen

$\mathrm{Cpo}=$ desired concentration

$\mathrm{K}_{10}=$ elimination constant

$\mathrm{V}_{1}=$ central compartment volume

$\left[\mu \mathrm{g} \cdot \mathrm{kg}^{-1} \cdot \mathrm{min}^{-1}\right]$
$\left[\mu \mathrm{g} \cdot \mathrm{ml}^{-1}\right]$
$\left[\mathrm{min}^{-1}\right]$
$[\mathrm{l}]$

To prevent a concentration $x$ time curve drop, Bailey ${ }^{9}$ proposes an initial bolus followed by continuous infusion for 30 minutes. The infusion should be then adjusted at 1 hour intervals (30-90 min, 90-150 min, 150-210 min etc).

In fact, the numeric calculation program is executed based on the three compartments model which calculates infusion rates needed for the concentrations to reach a predetermined value in $30 \mathrm{~min}, 90$ minutes and subsequent hours (Tables II and III). In general, infusion rate is decreased in every stage but, depending on the initial bolus dose, it is possible that infusion may have to be increased from the first to the second stage.
Actually, the time in which the infusion rates are fixed could be any, and if stage intervals were decreased, concentration would deviate less from the target. Computer emulations generate results as "precise" as those obtained by Marsh ${ }^{13}$ and Short ${ }^{14}$.

The results obtained should be seen with some criticism, given the limitations of the three compartments model itself. Drug predicted blood concentration obtained by calculation is not exactly in agreement with patients' actual blood concentration. This is due firstly to pharmacokinetic parameters obtained from measurements in a very small population. Statistically, one cannot assure that those samples are fully representative of the universe of individuals. Even if parameters are normalized by weight, gender and age, clinical status and physiological differences may vary ${ }^{24}$. Moreover, even if drug blood concentration is exactly the same as the calculated one, concentration needed for a given response to a stimulation differs from patient to patient. On the other hand, the technique is justified because potential blood concentration deviations wouldn't go beyond drug's therapeutic window limits. That what was observed in this study: hemodynamic parameters and hypnotic levels were similar in both groups. This study also showed that individual variations occurred, part of the reason why collected data were different from those computer accurately calculated.

Another interesting result was similar BIS-evaluated hypnosis levels observed in both groups, showing that different propofol infusions determined by pharmacokinetic programs produce concentrations within the same therapeutic window. It must be remembered, however, that median BIS values were lower in Group S, with the exception of M5, which was influenced by the initial propofol bolus. These results are original, and there is no pediatric paper in the literature studying $\mathrm{BIS}$ related to propofol blood concentration (Table VI and Figure 1). There is one study evaluating hypnosis levels in children under sevoflurane ${ }^{25,26}$ or sevoflurane associated to $\mathrm{N}_{2} \mathrm{O}^{27}$.

There is no method able to compare analgesic depth between groups. The only data showing that groups were similar in this aspect was the inexistence of significant difference in alfentanil bolus administration. This complementation was minimal and restricted to 4 Group S and 5 Group M patients. This demonstrates the analgesic efficacy of $60 \% \mathrm{~N}_{2} \mathrm{O}$ associated to propofol.

This study has used, in an original manner, pharmacokinetic parameters obtained from two researches with children aged 4 to 12 years ${ }^{13,14}$. For such, a software (PROCRIV, PROCHIV) was employed, based on a Bailey's study ${ }^{9}$ proposing a simplified method to maintain a drug blood concentration approximately constant. The technique was developed to enable anesthesiologists to maintain a desired target blood concentration approximately constant. After an arbitrary bolus dose, decreasing infusion rates are calculated to maintain a stable blood level. These infusion rates are obtained from equations which calculate sequential infusion rate schedules. The accuracy of this technique was measured by Error Performance Median (EPM). It is an interna- 
tional consensus to consider acceptable for clinical use programs using pharmacokinetic parameters where EPM is equal to or lower than 30 . EPM results in our study showed that median levels of 8.4 in moment 20 for the Marsh group. After this, EPM increased to 16 in moment 40 and decreased to 4.6 in moment 60 , showing that, after 1 hour, predicted concentration had come close to measured concentration. Short program ${ }^{14}$ had an opposite behavior: EPM was 16 at 20 minutes, followed by 12.1 and 52 at 40 and 60 minutes of propofol infusion (Table VII and Figure 4). Our results have shown that Marsh's ${ }^{13}$ pharmacokinetic parameters had a good performance and remained within limits of \pm 30 . When predicted and measured concentrations were compared through Error Performance Absolute Median (EPAM), absolute values of 28.7 in moment $20,33.2$ in moment 40 and 23.6 in moment 60 were obtained for Group M; for Group S those values were 44.7 in moment $20,36.3$ in moment 40 and 53.8 in moment 60 . These results have shown that during 1 hour of propofol infusion there was an uniformity of results with Marsh's pharmacokinetic parameters ${ }^{13}$, while in Group S there was a cumulative effect, reflecting in a statistically significant difference between groups in moment 60 .

Our major objective was to evaluate whether a pharmacokinetic parameter for propofol infusion associated to $\mathrm{N}_{2} \mathrm{O}$ in children could significantly influence hypnosis and anesthesia (Table VI and Figure 1). There were no significant differences in hemodynamic parameters (systolic and diastolic blood pressure and hear rate) for both groups. $\mathrm{SpO}_{2}$, nitrous oxide inspired concentration $\left(\mathrm{FiN}_{2} \mathrm{O}\right)$ and $\mathrm{P}_{\mathrm{ET}} \mathrm{CO}_{2}$ showed also no significant difference among moments and between groups. Also, the amount of additional analgesics was similar between groups, showing that pharmacokinetic programs are clinically equivalent. This result is understandable because, even with pharmacokinetic differences, target blood concentrations associated to $\mathrm{N}_{2} \mathrm{O}$ remained within the therapeutic window. In our study, pharmacokinetic parameters were obtained from pediatric patients from two continents (Europe and Asia) and applied to Brazilian children. It is more likely that Short's pharmacokinetic program, based on Hong-Kong children, has overestimated Brazilian pediatric patients' target blood concentration. Conversely, Marsh's pharmacokinetic parameters obtained from European children, came closer to $4 \mu \mathrm{g} \cdot \mathrm{ml}^{-1}$ propofol target concentration in our patients.

Clinically, patients had similar behaviors, when accessed three a more precise technique to evaluate hypnosis, like the microprocessed EEG equipment - BIS (Table VI and Figure 1). From the laboratorial point of view, by measuring propofol blood concentration, this similarity was not seen (Tables VII, VIII and IX, Figures 2, 3 and 4).

Concluding, the comparison between Marsh's ${ }^{13}$ and Short's ${ }^{14}$ parameters has shown that both computer programs can be used in pediatrics patients, but our preference is for PROCHIV-Marsh program for using less propofol associated to $\mathrm{N}_{2} \mathrm{O}$, for reaching a more accurate target concentration and inducing adequate hypnosis levels in children aged 4 to 12 years.

\section{REFERÊNCIAS - REFERENCES}

01. Kruger-Thiemer E - Continuous intravenous infusion and multicompartiment accumulation. Eur J Pharmacol, 1968;4: 317-324.

02. Hull CJ - Pharmacokinetics for Anesthesia. Oxford: ButterworthHeinemann, 1991;124-126.

03. Shafer A, Doze VA, Shafer SL et al - Pharmacokinetics and pharmacodynamics of propofol infusions during general anesthesia. Anesthesiology, 1988;69:348-356.

04. Glass PSA, Shafer SL, Reves JG - Intravenous Drug Delivery Systems, em: Miller RD - Anesthesia. $5^{\text {th }}$ Ed, New York, Churchill Livingstone, 2000;377-411.

05. Shafer SL, Toward S - Optimal intravenous dosing strategies. Semin Anesth, 1993;12:222-234.

06. Jacobs JR, Reves JG, Glass PSA - Rationale and technique for continuous infusions in anesthesia. Int Anesthesiol Clin, 1991; 29:23-38.

07. Reves JG, Jacobs J, Glass PSA - Automated drug delivery in anesthesia. Semin Anesth, 1991;12:127-137.

08. Browne BL, Prys-Robert C, Wolf AR - Propofol and alfentanil in children: infusion technique and dose requirement for total I.V. anaesthesia. Br J Anaesth, 1992;69:570-576.

09. Bailey JM - A technique for approximately maintaining constant plasma levels of intravenous drugs. Anesthesiology, 1993;78: 116-123.

10. Vianna PTG, Fernandes Filho GEF - O microcomputador como coadjuvante da anestesia intravenosa contínua. Rev Bras Anestesiol, 1993;43(Sup17):CBA59.

11. Vianna PTG, Fernandes Filho GEF, Ganem EM et al - Método simplificado para manutenção constante da concentração plasmática de propofol em nível aproximadamente constante em pacientes pediátricos. Rev Bras Anestesiol, 1995;45 (Supl19):CBA72.

12. Vianna PTG, Fernandes Filho GEF, Gusman P et al - Método simplificado para manutenção da concentração plasmática de sufentanil em nível aproximadamente constante. Rev Bras Anestesiol, 1996;46:249-258.

13. Marsh $\mathrm{B}$, White $\mathrm{M}$, Morthon $\mathrm{N}$ et al - Pharmacokinetic model driven infusion of propofol in children. $\mathrm{Br} \mathrm{J}$ Anaesth, 1991;67: 41-48.

14. Short TG, Aun CST, Tan P et al - A prospective evaluation of pharmacokinetic model controlled infusion of propofol in paediatric patients. Br J Anaesth, 1994;72:302-306.

15. Vianna PTG, Vane LA - Anestesia venosa total contínua método simplificado de quantificação de doses. Rev Bras Anestesiol, 1990;40:343-345.

16. Rampil IJ - A primer for EEG signal processing in anesthesia. Anesthesiology, 1998;89:980-1002.

17. Plummer $\mathrm{G}$ - Improved method for the determination of propofol in blood by high performance liquid chromatography with fluorescence detection. J Chromatogr, 1987;421:171-176.

18. Morrison DF - Multivariate Statistical Methods. $3^{\text {rd }}$ Ed, New York, McGraw Hill, 1990;1-495.

19. Fisher LDV, Belle G - Biostatistics: A Methodology for the Health Sciences. New York, Wiley-Interscience, 1993;1-991.

20. Smith I, White PF, Nathanson M et al - Propofol: an update on its clinical use. Anesthesiology, 1994;81:1005-1043.

21. Jones RDM, Chan K, Andrew LJ - Pharmacokinetics of propofol in children. Br J Anaesth, 1990;65:661-667.

22. Kataria BK, Ved SA, Nicodemus HF et al - The pharmakinetics of propofol in children using three different data analysis approaches. Anesthesiology, 1994;80:104-122. 
23. Maitre PO, Shafer SL - A simple pocket calculator approach to predict anesthetic drug concentration from pharmacokinetic data. Anesthesiology, 1990;73:332-336.

24. Thompson IA, Fitch W, Hughes RL et al - Effects of certain intravenous anaesthetic on liver blood flow and hepatic oxygen consumption in the greyhound. Br J Anaesth, 1986;58:69-80.

25. Watcha MF, Galinkin J, Fazi LM et al - Bispectral index (BIS) EEG monitoring in children. Anesthesiology; 1999;91:A1264.

26. Ganem EM, Módolo NSP, Vianna PTG et al - Titulação do sevoflurano utilizando o índice bispectral em anestesia pediátrica. Influência da medicação pré-anestésica no tempo de despertar e no consumo de anestésico inalatório. Rev Bras Anestesiol, 2000;50:197-201.

27. Bannister CF, Brosius KK, Sigl JC et al - Effect of bispectral index monitoring on anesthetic use and recovery in children anesthetized with sevoflurane in nitrous oxide. Anesth Analg, 2001;92:877-881.

\section{RESUMEN}

Vianna PTG, Vilela EP, Cordon FCO, Carvalho LR - Método Simplificado para Manutención de la Concentración Sanguínea de Propofol en Nivel Aproximadamente Constante, Asociado al Óxido Nitroso en el Paciente Pediátrico

Justificativa y Objetivos - La manutención de concentración sanguínea alvo-controlada en niveles aproximadamente constantes del propofol es una técnica que puede ser empleada de modo simplificado en la sala de cirugía. La finalidad de esta pesquisa es comparar clínica y laboratorialmente la infusión de propofol en niños usando los atributos farmacocinéticos de Short y de Marsh.

Método - Fueron estudiados 41 pacientes con edades entre $4 y$ 12 años, de ambos sexos, estado físico ASA I ó II, distribuidos en dos grupos S (20 pacientes) y M (21 pacientes). En el Grupo
S se utilizaron los atributos farmacocinéticos de Short, y en el Grupo M, los atributos farmacocinéticos de Marsh. La inducción anestésica fue hecha con bolus de alfentanil 30 $\mu \mathrm{g} \cdot \mathrm{kg}^{-1}$, propofol $3 \mathrm{mg} \cdot \mathrm{kg}^{-1}$ y pancuronio, $0,08 \mathrm{mg} \cdot \mathrm{kg}^{-1}$ por vía venosa. Se procedió a intubación traqueal y a manutención con $\mathrm{N}_{2} \mathrm{O} / \mathrm{O}_{2}(60 \%)$ en ventilación controlada mecánica. En el grupo S la infusión de propofol fue de 254 (30 min) seguido de 216 $\mu \mathrm{g} \cdot \mathrm{kg}^{-1} \cdot \mathrm{min}^{-1}$ por más $30 \mathrm{~min}$. En el grupo $\mathrm{M}$ la infusión de propofol fue de 208 (30 min) seguido de $170 \mu \mathrm{g} \cdot \mathrm{kg}^{-1} \cdot \mathrm{min}^{-1}$ por más 30 min. A través del atributo farmacocinético específico a cada grupo la meta fue la obtención de la concentración-alvo de $4 \mu \mathrm{g} \cdot \mathrm{kg}^{-1}$ de propofol. Fueron cogidas tres muestras sanguíneas (a los 20, 40 y 60 minutos) para la dosificación del propofol por el método de la Cromatografia Líquida de Alta Performance.

Resultados - Los Grupos S y M fueron considerados similares cuanto a la edad, altura, peso y sexo $(p>0,05)$. No hubo diferencia estadística significativa entre los dos grupos estudiados para los parámetros: PAS, PAD, FC, FiN ${ }_{2} \mathrm{O}, \mathrm{SpO}_{2}$ de la hemoglobina y $\mathrm{P}_{E T} \mathrm{CO}_{2}$ al final de la expiración. La comparación entre grupos en número de bolus repetidos de alfentanil no fue estadísticamente significativa. El índice bispectral (BIS), no presentó diferencia estadísticamente significativa entre $M_{0}$ (vigilia) y los demás momentos en ambos grupos. Los valores Medianos de la Performance de Error (MPE) y los valores Medianos Absolutos de la Performance de Error (MAPE) mostraron diferencias estadísticas significativas entre los grupos en el momento 60. Valores medianos de la concentración sanguínea de propofol $\left(\mu \mathrm{g} \cdot \mathrm{kg}^{-1}\right)$ mostraron diferencias estadísticas significativas entre $M$ y $S$ en el momento 60 y entre el momento 40 y 60 en el grupo $S$.

Conclusiones - La anestesia con propofol usando los atributos farmacocinéticos de Marsh (Grupo M) presentó menor error en el cálculo de la concentración-alvo de propofol de $4 \mu \mathrm{g} \cdot \mathrm{kg}^{-1}$. Además de eso, utiliza menor cantidad de propofol para obtener resultados clínicos semejantes. Por todas esas calidades debe ser el preferido para uso en niños ASA I y con edades entre 4 y 12 años. 